\title{
Barley Transformation by Particle Bombardment Using Callus
}

\author{
Sung-Yong Kim*
}

Department of Plant Breeding, Swedish University of Agricultural Sciences, Alnarp, Sweden *For correspondence: sung.yong.kim@slu.se

\begin{abstract}
[Abstract] The delivery of desired construct into plant cells relies mainly on Agrobacterium-mediated transformation. However, Agrobacterium transformation is still very challenging for barley as only one genotype is amendable to Agrobacterium infection. The demand for developing genotype independent transformation methods is thus high. Here we report a transformation protocol for barley cultivar "Rika" by particle bombardment.
\end{abstract}

Keywords: Barley, Particle bombardment, Immature embryo, Transformation

[Background] Tissue culture based Agrobacterium transformation is commonly used for introducing vector DNAs into plant cells, but it is often genotype dependent. The availability of an efficient transformation protocol is thus crucial for the success of genome editing via Agrobacterium transformation. Barley is one of the economically important cereal crops, unfortunately, Agrobacterium transformation is in principle only working for the cultivar 'Golden Promise'. Developing alternative transformation methods is thus required to facilitate genetic improvement by genome editing of the crop. Particle bombardment can deliver vector DNAs into plant cells directly, which could be an alternative transformation method for barley. Here we report a particle bombardment protocol for barley transformation modified according to wheat particle bombardment using callus (Tian et al., 2019).

\section{Materials and Reagents}

1. $0.6 \mu \mathrm{m}$ gold particle (Bio-Rad, catalog number: 1652262)

2. Macrocarrier disk (Bio-Rad, catalog number: 1652335)

3. Stopping screen (Bio-Rad, catalog number: 1652336)

4. 1,350 psi rupture disk (Bio-Rad, catalog number: 1652330)

5. Micropore tape (3M, catalog number: 1530-1)

6. Petri dish

7. Eppendorf tube

8. Barley cv. Rika seeds

9. Calcium chloride (Sigma-Aldrich, catalog number: C1016-100G)

10. Sodium hypochlorite, $6-14 \%$ (Sigma-Aldrich, catalog number: $425044-250 \mathrm{ML}$ )

11. Spermidine (Sigma-Aldrich, catalog number: S2626)

12. Isopropanol (Sigma-Aldrich, catalog number: I19516-500ML)

13. MS medium (Duchefa, catalog number: M0245) 
14. Casein hydrolysate (Duchefa, catalog number: C1301)

15. $\mathrm{CuSO}_{4} \cdot 5 \mathrm{H}_{2} \mathrm{O}$ (Sigmal-Aldrich, catalog number: $451657-10 \mathrm{G}$ )

16. Sucrose (Duchefa, catalog number: S0809)

17. Maltose (Duchefa, catalog number: M0811)

18. Glutamine (Duchefa, catalog number: G0708)

19. Proline (Duchefa, catalog number: P0717)

20. Dicamba (Duchefa, catalog number: D0920)

21. Ascorbic acid (Duchefa, catalog number: A0602)

22. Zeatin riboside (Duchefa, catalog number: Z0937)

23. Gelrite (Duchefa, catalog number: G1101)

24. Ethanol

25. Plasmid DNA (see Recipes)

26. Gold particle stock solution (see Recipes)

27. Calcium chloride stock solution $\left(2.5 \mathrm{M}, \mathrm{CaCl}_{2}\right)$ (see Recipes)

28. Spermidine stock solution ( $0.1 \mathrm{M})$ (see Recipes)

29. Callus induction medium (see Recipes)

30. Shoot induction medium (see Recipes)

31. Potassium hydroxide solution (1 M) (see Recipes)

\section{Equipment}

1. Biolistic PDS-1000/He Particle delivery system (Bio-Rad, model: 165-2257)

2. Welch ILMVAC Rotary vane vacuum pump (Fisher Scientific)

3. Helium gas tank (AGA, Sweden).

4. Centrifuge (Eppendorf, model: 5417R)

5. Stereo microscope (Olympus)

6. Climate chamber

7. Laminar flow hood

\section{Procedure}

A. Callus induction

1. The seeds of spring barley cv. Rika are sown in soil (Emmaljunga, \# 1659) in $5 \mathrm{~L}$ pots and grown in a well-controlled climate chamber with speed breeding conditions: temperature $22 / 20{ }^{\circ} \mathrm{C}$ (day/night), day length $20 \mathrm{~h}$, humidity $80 \%$, and light intensity $500 \mu \mathrm{mol} / \mathrm{m}^{-2} / \mathrm{s}^{-2}$ at plant level using metal-halogen lamps (Ghosh et al., 2018).

2. Immature seeds are collected at 11-14 days after flowering and trimmed by hand (Figure 1A).

3. The seeds are surface sterilized in $70 \%(\mathrm{v} / \mathrm{v})$ ethanol for $2 \mathrm{~min}$, then in $10 \%$ sodium hypochlorite solution for 4 min (Recipe 4), followed by three rinses with sterilized $\mathrm{H}_{2} \mathrm{O}$. 
4. The immature embryos (size range $0.5-1.5 \mathrm{~mm}$ ) are excised under stereo microscope in a laminar flow hood using two forceps. The excised embryos are placed with the scutellum side up on the callus induction medium (Recipe 6). For each bombardment, about 10-13 embryos are placed in the center of the Petri dish with the embryos close, but no touching with each other (Figure 1B).

5. The excised embryos are grown in Petri dishes in a climate chamber for 10 to 14 days for callus induction and growth (Figure 1C). The conditions of the chamber are $25^{\circ} \mathrm{C} / 18{ }^{\circ} \mathrm{C}$ (day/night) without any light.

Note: It is important to ensure a good callus induction prior to particle bombardment.
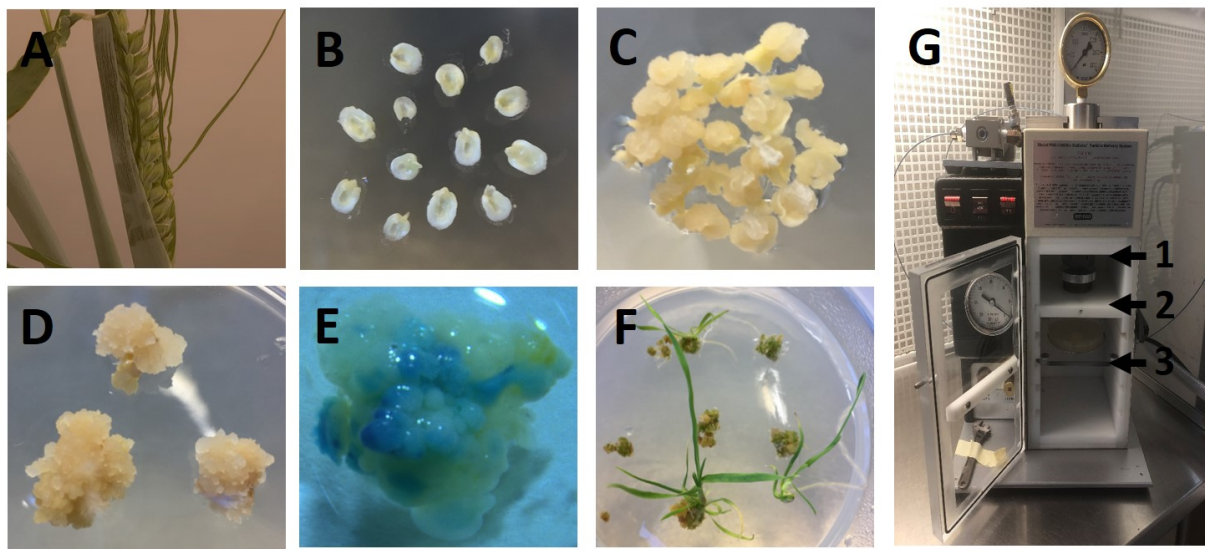

Figure1. Plant regeneration from Barley 'Rika' immature embryos after bombardment and bombardment chamber assembly. A. Barley seeds are ready for exising immature embryos. B. Immature embryos with scutellum side up excised from the sterile growing seeds. C. Ten days after culture on callus induction medium. D. One month after culture on callus induction medium. E. Embryogenic calli bombarded with a plasmid containing the GUS gene, showing blue color one week after bombardment. F. Shoots regenerated from the bombarded calli on shoot induction medium. G. Biolistic bombardment chamber, showing 1: the Rupture disk detaining cap, 2: macrocarrier launch assembly and 3: target shelf.

B. DNA coating onto gold particles

1. Add $5 \mu \mathrm{l}$ plasmid DNA and $50 \mu \mathrm{l}$ gold particle solution into a $1.5 \mathrm{ml}$ Eppendorf tube (E-tube) (Recipes 1-2).

2. Gently mix the solutions by pipetting.

3. Add $20 \mu \mathrm{l}$ calcium chloride stock solution (Recipe 3) onto the E-tube lid.

4. Add $50 \mu \mathrm{l}$ spermidine stock solution (Recipe 5) into calcium chloride stock solution on the Etube lid and mix well.

Note: Do not mix calcium chloride and spermidine stock solutions prior to addition of plasmid $D N A$.

5. Close the E-tube and mix the solutions well by tapping by hand or vortexing for $1 \mathrm{~min}$ (Longer vortexing may cause particle aggregation). 
6. Put the E-tube on ice for $1 \mathrm{~min}$.

7. Centrifuge at $1,957 \times g$ for $30 \mathrm{~s}$.

8. Remove the supernatant without touching the gold particles.

9. Wash the gold particles with $250 \mu \mathrm{l} 99 \%$ ethanol and mix well by pipetting or vortexing (Make sure to re-suspend the particles in ethanol without clump).

10. Centrifuge at $1,957 \times g$ for $30 \mathrm{~s}$ and remove supernatant.

11. Re-suspend the gold particles well with $60 \mu \mathrm{l} 99 \%$ ethanol by pipetting or vortexing. Note: It is better to use DNA coated gold particles right away.

C. Particle bombardment procedure

1. Check the machine prior to particle bombardment

a. Turn on the machine and open the helium gas tank valve.

b. Soak the 1,350 psi rupture disk in isopropanol and place the disk onto the detaining cap.

c. Tighten the retaining cap using Bio-Rad torque wrench.

d. Close the bombardment chamber.

e. Turn on the vacuum pump and hold it at 28 vacuum gauge.

f. Hold the Fire button until the rupture disk burst out at 1,350 psi while checking the psi gauge on the pressure meter.

g. Turn off the vacuum pump and wait until the chamber is de-pressurized.

2. Particle bombardment under sterile conditions

All operations from now on will be carried out in a laminar flow hood under sterile conditions. Clean the entire bombardment chamber and the needed materials with $70 \%$ ethanol first whenever applicable.

a. Soak the macrocarrier and stopping screen in $70 \%$ ethanol and let them dry.

b. Mix well the DNA-coated gold particles prior to use and load $5 \mu \mathrm{l}$ of the particles evenly in the center of the macrocarrier disk and let them dry.

The gold particles could hardly be seen if they are evenly distributed on the disk. Prepare macrocarrier immediately prior to use otherwise the disk will be dried out due to quick evaporation of ethanol (Recipe 2).

c. Soak the 1,350 psi rupture disk in isopropanol and place the rupture disk into the detaining cap.

d. Tighten the rupture disk retaining cap using the Bio-Rad torque wrench.

e. Place the stop screen in the sample block.

f. Put the macrocarrier disk with the gold particles' side down to the macrocarrier ring using the seating tool.

g. Fasten the macrocarrier ring with cover. Place the macrocarrier launch assembly on the $1^{\text {st }}$ shelf of the bombardment chamber (Figure 1F).

h. Put a Petri dish with the pre-cultured barley embryos without lid on the target plate, which is placed on the $3^{\text {rd }}$ shelf in the bombardment chamber (Figure $1 \mathrm{~F}$ ). 
i. Close the chamber door.

j. Turn on the vacuum pump and hold vacuum gauge at 28 .

k. Press and hold the Fire button until a "pop" sound is heard at 1,350 psi while checking the helium gas pressure gauge.

I. Turn off the vacuum pump and wait until the chamber is de-pressurized.

m. Take out the Petri dish, put the lid on and tape it with micropore tape.

$\mathrm{n}$. The barley plate is then covered with aluminum foil and placed in the growth chamber with the conditions as stated above.

D. Culture growth, shoot regeneration and planting in soil

1. The bombarded embryos with calli are grown in the growth chamber for 2 days and transferred onto antibiotic contained callus induction media $(20 \mu \mathrm{g} / \mathrm{ml}$ in case of hygromycin) until shoots are formed, which will take 3 or 4 weeks or even longer.

2. Check for any contamination every 3 or 4 days during the first two weeks.

3. Transfer embryos with regenerated shoots to the shoot induction medium with an appropriate antibiotic selection. The barley plate is needed to cover with a paper to prevent photo-damage for first two days after transferring on shoot induction media (Recipe 7).

4. Maintain the shoot cultures in the growth chamber until the roots are formed, which will take around 2-3 weeks.

5. Plant the plantlets in soil.

\section{Recipes}

1. Plasmid DNA

Plasmid DNA is prepared upon user's preference protocol and appropriate plasmid DNA concentration is $1 \mu \mathrm{g} / \mu \mathrm{l}$

2. Gold particle stock solution

a. Weight $40 \mathrm{mg}$ gold particles in an Eppendorf tube and add $1 \mathrm{ml}$ of $99 \%$ ethanol

b. Mix well by spinning down

c. Remove the supernatant

d. Add $1 \mathrm{ml}$ of $\mathrm{ddH}_{2} \mathrm{O}$ and mix well. Aliquot $50 \mu \mathrm{l}$ into sterile Eppendorf tubes and keep them at $-20{ }^{\circ} \mathrm{C}$ until use

3. Calcium chloride stock solution $\left(2.5 \mathrm{M}, \mathrm{CaCl}_{2}\right)$

Weigh $55.49 \mathrm{~g}$ of $\mathrm{CaCl}_{2}$ and dissolve the powder by adding $200 \mathrm{ml} \mathrm{H}_{2} \mathrm{O}$

4. Spermidine stock solution $(0.1 \mathrm{M})$

a. Weigh $0.145 \mathrm{~g}$ of spermidine and dissolve the powder in a tube by adding $10 \mathrm{ml} \mathrm{H}_{2} \mathrm{O}$

b. Keep spermidine stock solution at $-80^{\circ} \mathrm{C}$. Make new stocks every six month

5. Callus induction medium

$4.3 \mathrm{~g} / \mathrm{L}$ full strength of MS medium 
$100 \mathrm{mg} / \mathrm{L}$ casein hydrolysate

$2.5 \mathrm{mg} / \mathrm{L}$ dicamba

$100 \mathrm{mg} / \mathrm{L}$ ascorbic acid

$1.25 \mathrm{mg} / \mathrm{L} \mathrm{CuSO}_{4} \cdot 5 \mathrm{H}_{2} \mathrm{O}$

$10 \%$ sucrose

$20 \%$ maltose

$500 \mathrm{mg} / \mathrm{L}$ glutamine

$500 \mathrm{mg} / \mathrm{L}$ proline

$6 \mathrm{~g} / \mathrm{L}$ Gelrite

Adjust pH 5.7 using $1 \mathrm{M} \mathrm{KOH}$

6. Shoot induction medium

Shoot induction medium is the same as callus induction medium except that dicamba is replaced with $5 \mathrm{mg} / \mathrm{L}$ zeatin riboside

7. Potassium hydroxide solution (1 M)

a. Weigh $56.11 \mathrm{~g}$ of potassium hydroxide and dissolve the powder in $900 \mathrm{ml} \mathrm{H}_{2} \mathrm{O}$

b. Adjust volume to $1,000 \mathrm{ml}$ by adding $\mathrm{H}_{2} \mathrm{O}$ and Keep at room temperature

\section{Acknowledgments}

The protocols was adapted from Harwood and Smedley (2009) and Tian et al. (2019) with modifications. This work is partially financed by Einar and Inga Nilssons Foundation.

\section{Competing interests}

The author have no conflicts of interest to declare.

\section{References}

1. Ghosh, S., Watson, A., Gonzalez-Navarro, O. E., Ramirez-Gonzalez, R. H., Yanes, L., Mendoza-Suarez, M., Simmonds, J., Wells, R., Rayner, T., Green, P., Hafeez, A., Hayta, S., Melton, R. E., Steed, A., Sarkar, A., Carter, J., Perkins, L., Lord, J., Tester, M., Osbourn, A., Moscou, M. J., Nicholson, P., Harwood, W., Martin, C., Domoney, C., Uauy, C., Hazard, B., Wulff, B. B. H. and Hickey, L. T. (2018). Speed breeding in growth chambers and glasshouses for crop breeding and model plant research. Nat Protoc 13(12): 2944-2963.

2. Harwood, W. A. and Smedley, M. A. (2009). Barley transformation using biolistic techniques. In Jones, H. D. and Shewry, P. R. (eds). Transgenic Wheat, Barley and Oats. Methods in Molecular Biology 478. Humana Press, New York, NY. 
3. Tian, B., Navia-Urrutia, M., Chen, Y., Brungardt, J. and Trick, H. N. (2019). Biolistic transformation of wheat. In: Kumar, S., Barone, P. and Smith, M. (Eds.). Transgenic Plants. Methods in Molecular Biology 1864. Humana Press, New York, NY. 